Original Research Paper

\title{
A Computational Method Based on Bernstein Polynomials for Solving FredholmIntegro-Differential Equations under Mixed Conditions
}

\author{
${ }^{1}$ Miloud Moussai and ${ }^{2}$ Lakhdar Chiter \\ ${ }^{I}$ Laboratoire de Mathématiques Pures et Appliquées, University of M'sila, M'sila, 28000 Algeria \\ ${ }^{2}$ Department of Mathematics, University of Setif, 19000 Algeria
}

Article history

Received: 21-10-2016

Revised: 27-01-2017

Accepted: 27-02-2017

Corresponding Author:

Miloud Moussai

Laboratoire de Mathématiques

Pures et Appliquées, University

of M'sila, M'sila, 28000

Algeria

Email:mi.moussai@gmail.com

\section{Introduction}

Integro-differential equations, which are composed by integral and differential equations, are a well-known mathematical tool and an important branch of modern mathematics. Among these equations, FredholmIntegroDifferential Equations (FIDE) are encountered in several areas such as biology, economics, engineering and many others, so as usual, there is no specific analytic method to solve this equations, several numerical methods are presented to approximate the solution of FIDEs. Recently, different methods based on basic functions have been proposed to approximate the solution of FIDE, such as orthogonal basis and wavelets. At the same time, various numerical methods take an important place in solving FIDE numerically, such as Legendre polynomials, which have been used for high-order linear FIDE (Yalçinbaş et al., 2009), rationalized Haar functions and Walsh series, differential transform method (Golubov et al., 1991) and many other known methods in the literature. Among these methods, the polynomials of Bernstein that have been widely used to solve both linear and non linear integro-differential equations. Very few paper using Bernstein polynomials to solve FIDE with mixed conditions have been considered in the literature.
The aim of this paper is to find the solution of the following integro-differential equation:

$$
\begin{aligned}
& Q(x) y^{\prime}(x)+R(x) y(x) \\
& +\lambda \int_{0}^{1} K(x, t) y(t) d t=f(x), 0 \leq x \leq 1
\end{aligned}
$$

under the following mixed conditions:

$$
\sum_{s=0}^{m} e_{s} r\left(r_{s}\right)=\mu \quad 0 \leq r_{s} \leq 1
$$

where, $Q, R, f$ and $K$ are continuous functions on the interval $[0,1]$. Equation 1.1 can be found in (IdreesBhattia and Bracken, 2007; Swarup, 2007).

This paper is organized as follows. In section 2, we introduce the properties of the Bernstein polynomials. In section 3, we present the numerical method to solve the problem (1.1-1.2). Illustrative examples are presented in section 4 and finally, in section 5 we give a brief conclusion.

\section{Properties of Bernstein Polynomials}

The Bernstein polynomials of degree $n$ on $[a, b]$ are defined by: 


$$
b_{i, n}(x)=\left(\begin{array}{l}
n \\
i
\end{array}\right) \frac{(x-a)^{i}(b-x)^{n-i}}{(b-a)^{n}}, i=0,1, \ldots, n
$$

where:

$$
\left(\begin{array}{l}
n \\
i
\end{array}\right)=\frac{n !}{i !(n-i) !}
$$

In what follows, we give some basic properties of the Bernstein polynomials. Formore details, we refer to (Isik et al., 2012; Boyer and Thiel, 2002):

$$
b_{i, n}(x)=0, \text { if } i<0 \text { or } i>n
$$

$\sum_{i=0}^{n} b_{i, n}(x)=1, \forall x \in[0,1], i=0,1, \ldots, n$

$b_{i, n}(a)=b_{i, n}(b)=0,1 \leq i \leq n-1$

In particular if $[a, b]=[0,1]$, we obtain:

$$
b_{i, n}(x)=\left(\begin{array}{l}
n \\
i
\end{array}\right) x^{i}(1-x)^{n-i}, 0 \leq x \leq 1, i=0,1,2, \ldots, n
$$

The linear combination of the basic Bernstein polynomials is given by the following formula:

$$
B(x)=\sum_{i=0}^{n} c_{i} b_{i, n}(x)
$$

where, $c_{i}$ are the Bernstein coefficients. We have also the following proprieties:

$$
\begin{gathered}
b_{i, n}(x) \geq 0, x \in[0,1](\text { positivity }) \\
b_{i . n}(x)=b_{n-i, n}(1-x)(\text { symmetry })
\end{gathered}
$$

and the recurrent formula:

$$
b_{i, n}(x)=\left\{\begin{array}{l}
(1-x) b_{i, n-1}(x), i=0 \\
(1-x) b_{i, n-1}(x)+x b_{i-1, n-1}, i=1, \ldots, n-1 \\
x b_{i-1, n-1}(x), i=n
\end{array}\right.
$$

The derivatives of different degrees of Bernstein polynomials have an important role in the numerical solution of differential and integro-differential equations.

\section{Lemma 2.1}

The derivative of order $k \geq 1$ of the Bernstein polynomials is given by:

$$
\begin{aligned}
& b_{i, n}^{(k)}(x) \\
& =\left(\prod_{s=0}^{s=k-1}(n-s)\right)\left(\sum_{m=0}^{m=k}(-1)^{m+2}\left(\begin{array}{l}
k \\
m
\end{array}\right) b(x)_{i-k+m, n-k}\right),(i \geq k \geq m)
\end{aligned}
$$

$$
b_{i, n}^{(k)}(x)=0 \text { if } n<k
$$

\section{Proof}

We prove the relation by induction. We check the property for $k=1$ :

$$
\begin{aligned}
& b_{i, n}^{\prime}(x)=\left(\prod_{s=0}^{s=0}(n-s)\right)\left(\sum_{m=0}^{m=1}(-1)^{m+2}\left(\begin{array}{l}
1 \\
m
\end{array}\right) b(x)_{i-1+m, n-1}(x)\right) \\
& b_{i, n}^{\prime}(x)=n\left(\left(\begin{array}{l}
1 \\
0
\end{array}\right) b_{i-1, n-1}(x)-\left(\begin{array}{l}
1 \\
1
\end{array}\right) b_{i, n-1}(x)\right) \\
& b_{i, n}^{\prime}(x)=n\left(b_{i-1, n-1}(x)-b_{i, n-1}(x)\right)
\end{aligned}
$$

Thus the relation holds for $k=1$.

It is assumed that the relation is true for $k \in N^{*}$ and we should prove it for $(k+1)$.

Indeed, we have:

$$
b_{i, n}^{(k+1)}(x)=\left[b_{i, n}^{(k)}(x)\right]^{\prime}
$$

$b_{i, n}^{(k)}(x)=\left(\prod_{s=0}^{s=k-1}(n-s)\right)\left(\begin{array}{l}b_{i-k, n-k}(x)-k b_{i-k+1, n-k}(x) \\ +\frac{k(k-1)}{2 !} b_{i-k+2, n-k}(x) \\ +\ldots+(-1)^{k+2} b(x)_{i, n-k}(x)\end{array}\right)$

$\left[b_{i, n}^{(k)}(x)\right]^{\prime}$

$=\left(\prod_{s=0}^{s=k-1}(n-s)\right)\left(\begin{array}{l}b_{i-k, n-k}^{\prime}(x)-k b_{i-k+1, n-k}^{\prime}(x) \\ +\frac{k(k-1)}{2 !} b_{i-k+2, n-k}^{\prime}(x) \\ +\ldots+(-1)^{k+2} b^{\prime}(x)_{i, n-k}(x)\end{array}\right)$

According to the above relations, we have:

$$
\begin{aligned}
& b_{i-k, n-k}^{\prime}(x)=(n-k) \quad\left(b_{i-k-1, n-k-1}(x) \quad-b_{i-k, n-k-1}(x)\right) \\
& b_{i-k+1, n-k}^{\prime}(x)=(n-k) \quad\left(b_{i-k, n-k-1}(x) \quad-b_{i-k+1, n-k-1}(x)\right) \\
& \text { ¿ } \quad \vdots \quad \vdots \\
& b_{i, n-k}^{\prime}(x)=(n-k) \quad\left(b_{i-k, n-k-1}(x) \quad-b_{i, n-k-1}(x)\right)
\end{aligned}
$$

Substituting (2.16) in the relation (2.15), we obtain: 


$$
\left[b_{i, n}^{(k)}(x)\right]^{\prime}=\prod_{s=0}^{s=k-1}(n-s)(n-k)\left(b(x)_{i-(k+1), n-k-1}-(k+1) b(x)_{i-k, n-(k+1)}+\frac{k(k-1)}{2 !} b(x)_{i-(k+1), n-(k+1)}+\ldots+(-1)^{k+2} b(x)_{i, n-(k+1)}\right)
$$

It is easy to check that:

$$
\begin{aligned}
& \left(\prod_{s=0}^{s=k-1}(n-s)\right)(n-k)=\left(\prod_{s=0}^{s=k}(n-s)\right) \\
& 1=\left(\begin{array}{c}
k+1 \\
0
\end{array}\right),(k+1)=\left(\begin{array}{c}
k+1 \\
1
\end{array}\right), \frac{k(k-1)}{2 !} \\
& =\left(\begin{array}{c}
k+1 \\
2
\end{array}\right), \ldots, 1=\left(\begin{array}{l}
k+1 \\
k+1
\end{array}\right)
\end{aligned}
$$

By substituting (2.18) and (2.19) in the relation (2.17), we obtain:

$$
\begin{aligned}
& {\left[b_{i, n}^{(k)}(x)\right]^{\prime}=\left(\prod_{s=0}^{s=k-1}(n-s)\right)\left(b(x)_{i-(k+1), n-k-1}\right.} \\
& -(k+1) b(x)_{i-k, n-(k+1)} \\
& +\frac{k(k-1)}{2 !} b(x)_{i-(k+1), n-(k+1)} \\
& \left.+\ldots+\left((-1)^{m+2}\right) b(x)_{i, n-(k+1)}\right) \\
& =\left(\prod_{s=0}^{s=k}(n-s)\right)\left(\left(\begin{array}{c}
k+1 \\
0
\end{array}\right) b(x)_{i-(k+1), n-k-1}\right. \\
& -\left(\begin{array}{c}
k+1 \\
1
\end{array}\right) b(x)_{i-k, n-(k+1)} \\
& +\left(\begin{array}{c}
k+1 \\
2
\end{array}\right) b(x)_{i-(k+1), n-(k+1)} \\
& \left.+\ldots+\left((-1)^{m+2}\right)\left(\begin{array}{l}
k+1 \\
k+1
\end{array}\right) b(x)_{i, n-(k+1)}\right) \\
& =\left(\prod_{s=0}^{s=k}(n-s)\right)\left(\sum_{m=0}^{m=k+1}(-1)^{m+2}\left(\begin{array}{c}
k+1 \\
m
\end{array}\right) b(x)_{i-(k+1)+m, n-(k-1)}\right) \\
& =b_{i, n}^{(k+1)}(x)
\end{aligned}
$$

which proves that the relation is true for all $k$.

\section{Approximation Function}

A function $f$ square integrable in the interval $[0,1]$ can be written as:

$f(t)=\lim _{n \rightarrow 0} \sum_{i=0}^{n} c_{i n} b_{i n}(t)$ where, $c_{i n}=\left\langle f, b_{i n}\right\rangle$ and $\langle$,$\rangle is the standard inner product on$ $L^{2}[0,1]$.

If the series (2.25) are trunctated at $n=m$, then we have (Isik et al., 2012):

$$
f(t)=\sum_{i=0}^{m} c_{i, m} b_{i, m}(t)=C^{T} B(t)
$$

where, $C$ and $B$ are $(m+1) \times 1$ matrices and are given by:

$$
C=\left[c_{0, m}, c_{1, m}, \ldots, c_{m, m}\right]^{T}
$$

and:

$$
B(t)=\left[b_{0, m}(t), b_{1, m}(t), \ldots, b_{m, m}(t)\right]^{T}
$$

\section{Numerical Method}

Consider the linear FIDE of the first order (1.1) with the mixed conditions (1.2). The approximation of $y(x)$ by the polynomials of Bernstein is given by:

$$
\begin{aligned}
& y(x)=\sum_{i=0}^{n} a_{i} b_{i, n}(x)=C^{T} B(x) \\
& C^{T}=\left(a_{0}, a_{1}, \ldots, a_{n}\right), B(x)=\left(b_{0, n}, b_{1, n}, \ldots, b_{n, n}\right)
\end{aligned}
$$

where, $b_{i, n}(x)$ are the Bernstein polynomials of degree $n$ defined in Equation 2.1 and $a_{i}(i=0,1, \ldots, n)$ are unknown parameters.

Derivating Equation 3.1, we get:

$$
y^{\prime}(x)=\sum_{i=0}^{n} n a_{i}\left(b_{i-1, n-1}(x)-b_{i, n-1}(x)\right)
$$

Substituting (3.2) into (1.1), we get:

$$
\begin{aligned}
& Q(x) \sum_{i=0}^{n} n a_{i}\left(b_{i-1, n-1}(x)-b_{i, n-1}(x)\right) \\
& +R(x) \sum_{i=0}^{n} a_{i} b_{i, n}(x)+\lambda \int_{0}^{1} K(x, t) \sum_{i=0}^{n} a_{i} b_{i, n}(t) d t=f(x)
\end{aligned}
$$

which can be written, after simplification, as:

$$
\left[\begin{array}{l}
R(x) b_{0, n}(x)-n Q(x) b_{0, n-1}(x) \\
+\lambda \int_{0}^{1} K(x, t) b_{0, n}(t) d t
\end{array}\right] a_{0}
$$


$+\sum_{i=1}^{n} a_{i}\left[\begin{array}{l}n Q(x)\left(b_{i-1, n-1}(x)-b_{i, n-1}(x)\right) \\ +R(x) b_{i, n}(x)+\lambda \int_{0}^{1} K(x, t) b_{i, n}(t) d t\end{array}\right]=f(x)$

Multiplying both sides in (3.4) by $b_{j, n}(x)$ and integrating with respect to $x$ from 0 to 1 we obtain:

$\int_{0}^{1}\left[\begin{array}{l}R(x) b_{0, n}(x)-n Q(x) b_{0, n-1}(x) \\ +\lambda \int_{0}^{1} K(x, t) b_{0, n}(t) d t\end{array}\right] a_{0} b_{J, n}(x) d x$

$+\sum_{i=1}^{n} a_{i} \int_{0}^{1}\left[\begin{array}{l}n Q(x)\left(b_{i-1, n-1}(x)-b_{i, n-1}(x)\right) \\ +R(x) b_{i, n}(x)+\lambda \int_{0}^{1} K(x, t) b_{i, n}(t) d t\end{array}\right] b_{J, n}(x) d x$

$+\int_{0}^{1} f(x) b_{J, n}(x) d x$

which can be written by the following system:

$$
\begin{gathered}
a_{0} \alpha_{J}+\sum_{i=1}^{n} a_{i} c_{i, J}=G_{J} \quad J=0,1, \ldots n \\
\alpha_{J}=\int_{0}^{1}\left[\begin{array}{l}
R(x) b_{0, n}(x)-n Q(x) b_{0, n-1}(x) \\
+\lambda \int_{0}^{1} K(x, t) b_{0, n}(t) d t
\end{array}\right] b_{J, n}(x) d x, J=0,1,2,3, \ldots, n \\
C_{i, J}=\int_{0}^{1}\left[\begin{array}{l}
n Q(x)\left(b_{i-1, n-1}(x)-b_{i, n-1}(x)\right) \\
+R(x) b_{i, n}(x)+\lambda \int_{0}^{1} K(x, t) b_{i, n}(t) d t
\end{array}\right] b_{J, n}(x) d x \\
G_{J}=\int_{0}^{1} f(x) b_{J, n}(x) d x, i=0,1, \ldots, n, J=0,1, \ldots, n-1
\end{gathered}
$$

Condition (1.2) can be written in the following form:

$$
\begin{aligned}
& \mu=\sum_{s=0}^{m} e_{s} y\left(r_{s}\right)=\sum_{s=0}^{m} e_{s} \sum_{i=0}^{n} a_{i} b_{i, n}\left(r_{s}\right) \\
& =\sum_{s=0}^{m} e_{s}\left(a_{0} b_{0, n}\left(r_{s}\right)+a_{1} b_{1, n}\left(r_{s}\right)+\ldots+a_{n} b_{n, m}\left(r_{s}\right)\right) \\
& =\left(\sum_{s=0}^{m} e_{s} b_{0, n}\left(r_{s}\right)\right) a_{0}+\left(\sum_{s=0}^{m} e_{s} b_{1, n}\left(r_{s}\right)\right) a_{1} \\
& +\ldots+\left(\sum_{s=0}^{m} e_{s} b_{n, m}\left(r_{s}\right)\right) a_{n}
\end{aligned}
$$

by setting:

$w_{k}=\left(\sum_{s=0}^{m} e_{s} b_{k, n}\left(r_{s}\right)\right), k=0, \ldots, n$

we obtain:

$$
\mu=w_{0} a_{0}+w_{1} a_{1}+\ldots+w_{n} a_{n}
$$

The general system can be obtained as follows:

$$
\left\{\begin{array}{l}
a_{0} \alpha_{J}+\sum_{i=1}^{n} a_{i} c_{i, J}=G_{J} \quad J=0,1,2, \ldots, n \\
\sum_{i=1}^{n} a_{i} w_{i}=\mu
\end{array}\right.
$$

and the linear algebraic equation is given by:

$$
\tilde{A} X=\tilde{b}
$$

where:

$$
\tilde{A}=\left(\begin{array}{cccc}
\alpha_{0} & C_{1,0} & \cdots & C_{n, 0} \\
\alpha_{1} & C_{1,1} & \cdots & C_{n, 1} \\
\vdots & \vdots & \ddots & \vdots \\
\alpha_{0} & C_{1, n-1} & \cdots & C_{n, n-1} \\
\omega_{0} & \omega_{1} & \cdots & \omega_{n}
\end{array}\right), X=\left(\begin{array}{c}
a_{0} \\
a_{1} \\
\vdots \\
a_{n-1} \\
a_{n}
\end{array}\right), \tilde{b}=\left(\begin{array}{c}
G_{0} \\
G_{1} \\
\vdots \\
G_{n-1} \\
\mu
\end{array}\right)
$$

The linear system corresponding to the solution of Equation 1.1 under the condition (1.2) is given by an approximate solution (Singh et al., 2009):

$$
\tilde{y}=a_{0} b_{0, n}(x)+a_{1} b_{1, n}(x)+\ldots+a_{n} b_{n, n}(x)
$$

\section{Remark 3.1}

The square error $E$ is defined by the formula:

$$
E=(y(x)-\tilde{y}(x))^{2}
$$

where, $y(x)$ and $\tilde{y}(x)$ are the exact and approximate solutions respectively.

\section{Illustrative Examples}

The following examples illustrate the efficiency of the used method. Note that in these examples, the series (3.1) are truncated at levels 4 and 5, i.e., $(n=4)$ and $(n=5)$.

\section{Example 4.1}

Consider the following linear FIDE equation of the first order with mixed conditions:

$$
\begin{aligned}
& \left\{\begin{array}{l}
x^{3} y^{\prime}(x)+e^{x} y(x)+2 \int_{0}^{1} e^{2(x+1) t-(x+1)} y(t) d t=g(x) \\
y(0)+y(1)=3.0860 \quad 0 \leq x \leq 1
\end{array}\right. \\
& g(x)=\left(2 x^{3}+e^{x}\right) e^{2 x-1}+\frac{e^{(x+2)}-e^{-(x+2)}}{x+2}
\end{aligned}
$$


The exact solution is given by:

$$
y(x)=e^{2 x-1}
$$

By a comparison of Equation 1.1 and 1.2 with the Equation 4.1, we find:

$$
\begin{aligned}
& Q(x)=x^{3}, R(x)=e^{x}, K(x, t)=e^{2(x+1) t-(x+1)}, \lambda=2 \\
& e_{0}=e_{1}=1, r_{0}=0, r_{1}=1, \mu=3.0860
\end{aligned}
$$

By using the method described in section (4), Equation 4.1 is then solved and we have:

$$
\begin{aligned}
& \alpha_{J}=\int_{0}^{1}\left[\begin{array}{l}
e^{x} b_{0, n}(x)-n x^{3} b_{0, n-1}(x) \\
+2 \int_{0}^{1} e^{2(x+1) t-(x+1)} b_{0, n}(t) d t
\end{array}\right] b_{J, n}(x) d x \\
& C_{i, J}=\int_{0}^{1}\left[\begin{array}{l}
n x^{3}\left(b_{i-1, n-1}(x)-b_{i, n-1}(x)\right) \\
+e^{x} b_{i, n}(x)+2 \int_{0}^{1} e^{2(x+1) t-(x+1)} b_{i, n}(t) d t
\end{array}\right] b_{J, n}(x) d x \\
& G_{J}=\int_{0}^{1}\left(\begin{array}{c}
\left(2 x^{3}+e^{x}\right) e^{2 x-1} \\
+\frac{e^{(x+2)}-e^{-(x+2)}}{x+2}
\end{array}\right) b_{J, n}(x) d x, J=0,1,2,3,4 \\
& w_{k}=b_{k, n}(0)+b_{k, n}(1), k=0,1 \ldots, 4
\end{aligned}
$$

For $n=4$, the matrix $\tilde{A}$ and the vector $\tilde{b}$ are given by:

$$
\tilde{A}=\left(\begin{array}{lllll}
0.1584 & 0.1266 & 0.1235 & 0.1441 & 0.1901 \\
0.0949 & 0.1350 & 0.1627 & 0.1911 & 0.2346 \\
0.0560 & 0.1095 & 0.1750 & 0.2428 & 0.3208 \\
0.0360 & 0.0758 & 0.1470 & 0.2712 & 0.4847 \\
1.0000 & 0.0000 & 0.0000 & 0.0000 & 1.0000
\end{array}\right), \tilde{b}=\left(\begin{array}{c}
0.9458 \\
1.1459 \\
1.4402 \\
1.8654 \\
3.0860
\end{array}\right)
$$

and:

$$
X=\left(a_{0}, a_{1}, a_{2}, a_{3}, a_{4}\right)^{T}
$$

Since $\operatorname{det} \tilde{A} \neq 0$; then equation $\tilde{A} X=\tilde{b}$ has a unique solution given by:

$$
X=(0.3743,0.5165,0.8987,1.3507,2.7118)^{T}
$$

The approximate solution is:

$$
\begin{aligned}
& \tilde{y}(x)=a_{0} b_{04}(x)+a_{1} b_{14}(x)+a_{2} b_{24}(x)+a_{3} b_{34}(x)+a_{4} b_{44}(x) \\
& \tilde{y}(x)=1.0093 x^{4}-0.6808 x^{3}+1.4404 x^{2}+0.5684 x+0.3743
\end{aligned}
$$

The graph of the exact and approximate solutions for Equation 4.1 is represented in Fig. 1. The results, together with the square error, are reported in Table 1 for different values of $x$.

\section{Example 4.2}

Consider the following linear FIDE of the first order with mixed conditions:

$$
\begin{aligned}
& \left\{\begin{array}{l}
\left(x^{2}+1\right) y^{\prime}(x)+x y(x)-2 \int_{0}^{1} e^{x+t} y(t) d t=g(x) \\
\frac{1}{\sqrt{e}} y\left(\frac{1}{2}\right)+y(1)=-0.6947 \quad 0 \leq x \leq 1
\end{array}\right. \\
& g(x)=\frac{\left(x^{2}+x+2-e^{2}\right) e^{x}}{2-e^{2}}
\end{aligned}
$$

The exact solution (4.2) is:

$$
y(x)=\frac{e^{x}}{2-e^{2}}
$$

We do the same as in example 1 by a comparison of the Equation 1.1 and 1.2 with (4.5), we find:

$$
\begin{aligned}
& Q(x)=x^{2}+1, R(x)=x, K(x, t)=e^{x+t}, \lambda=-2 \\
& \mu=0.6947, e_{0}=\frac{1}{\sqrt{2}}, e_{1}=1, r_{0}=\frac{1}{2}, r_{1}=1
\end{aligned}
$$

We solve (4.5) by using the method described in Section (4), for $(n=5)$, we find:

$$
\begin{aligned}
& a_{0}=-0.1855765, a_{1}=-0.2226918, a_{2}=-0.2690726, \\
& a_{3}=-0.327809, a_{4}=-0.4016988, a_{5}=-0.4947668
\end{aligned}
$$

The approximate solution is:

$$
\begin{aligned}
& \tilde{y}(x)=a_{0} b_{05}(x)+a_{1} b_{15}(x)+a_{2} b_{25}(x) \\
& +a_{3} b_{35}(x)+a_{4} b_{45}(x)+a_{5} b_{55}(x) \\
& \tilde{y}(x)=-0.00213837 x^{5}-0.0065302 x^{4}-0.0315287 x^{3} \\
& -0.0926552 x^{2}-0.1855765 x-0.1855765
\end{aligned}
$$

The graph of the exact and approximate solutions for Equation (4.5) is represented in Fig. 2 and the results are reported in Table 2 for different values of $x$. 
approximate solution $\quad+++++$

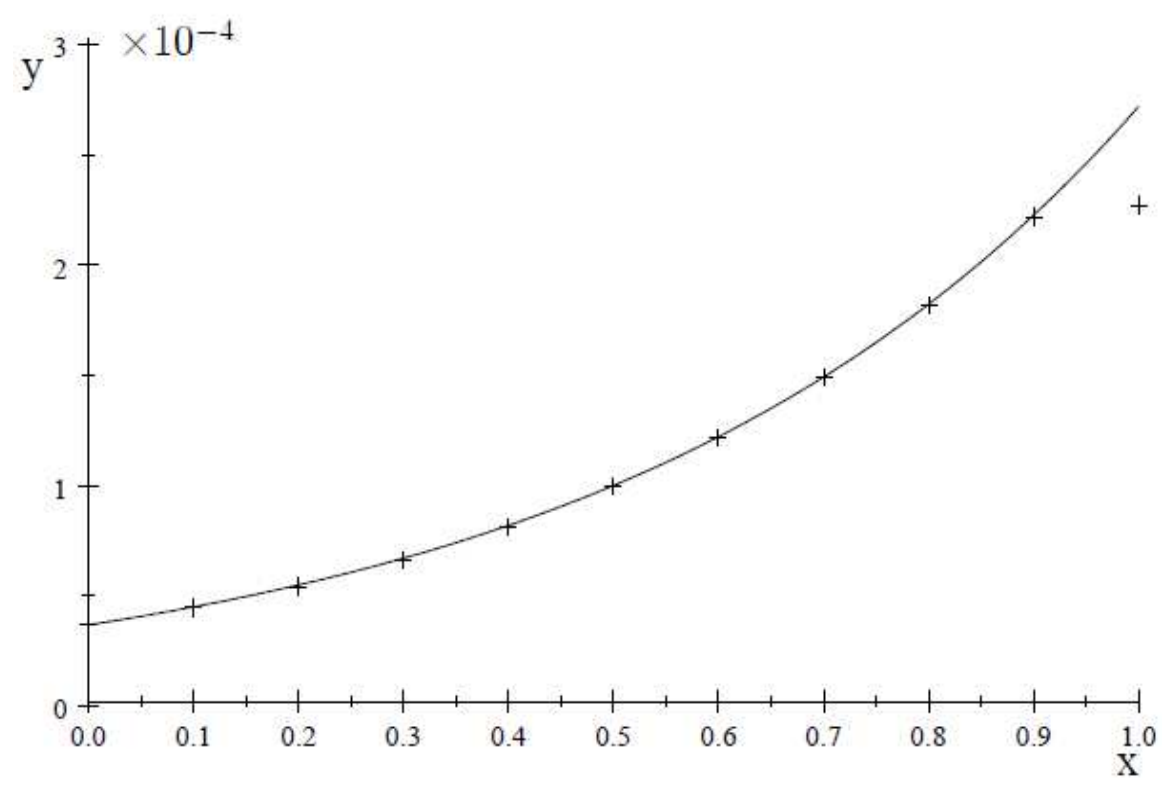

Fig. 1. Exact and approximate solution $(n=4)$ of example (4.1)

exact solution

approximate solution $\quad+++++$

$\times 10^{-4}$

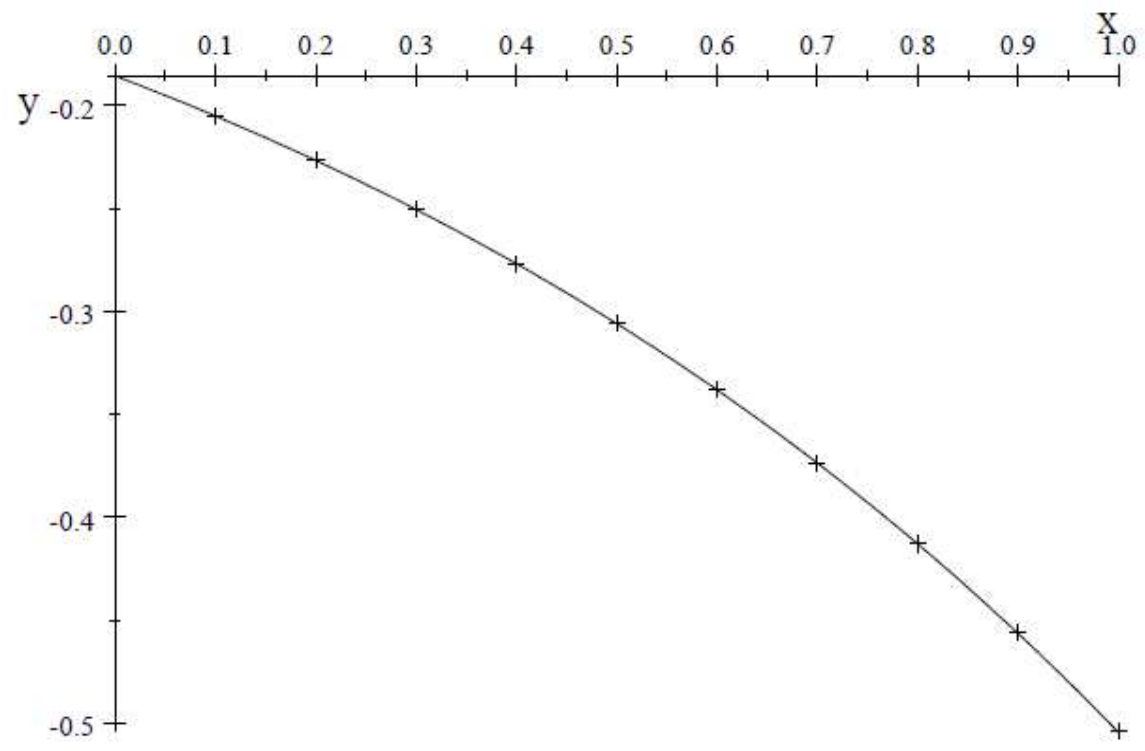

Fig. 2. Exact and approximate solutions for $(n=5)$ of example (4.2) 
Table 1. Exact and approximate solutions and square error for $(n=4)$, Example (4.1)

\begin{tabular}{llll}
\hline $\mathbf{x}$ & Exact solution & Approximate solution & Square error $(y(x)-\tilde{y}(x))^{2}$ \\
\hline 0 & 0.3679 & 0.3743 & $0.4096 \times 10^{-4}$ \\
0.1 & 0.4493 & 0.4451 & $0.1764 \times 10^{-4}$ \\
0.2 & 0.5488 & 0.5419 & $0.4761 \times 10^{-4}$ \\
0.3 & 0.6703 & 0.6643 & $0.1101 \times 10^{-4}$ \\
0.4 & 0.8187 & 0.8145 & $0.1764 \times 10^{-4}$ \\
0.5 & 1.0000 & 0.9967 & $0.1101 \times 10^{-4}$ \\
0.6 & 1.2214 & 1.2177 & $0.1369 \times 10^{-4}$ \\
0.7 & 1.4917 & 1.4869 & $0.2304 \times 10^{-4}$ \\
0.8 & 1.8221 & 1.8158 & $0.3469 \times 10^{-4}$ \\
0.9 & 2.2255 & 2.2186 & $0.4761 \times 10^{-4}$ \\
1 & 2.7183 & 2.7117 & $0.5476 \times 10^{-4}$ \\
\hline
\end{tabular}

Table 2. Exact and approximate solutions and square error for $(n=5)$ of Example (4.2)

\begin{tabular}{llll}
\hline $\mathrm{x}$ & Exact solution & Approximate solution & Square error $(y(x)-\tilde{y}(x))^{2}$ \\
\hline 0 & -0.1856 & -0.1856 & $0.0000 \times 10^{-4}$ \\
0.1 & -0.2051 & -0.2051 & $0.0000 \times 10^{-4}$ \\
0.2 & -0.2266 & -0.2267 & $0.0001 \times 10^{-4}$ \\
0.3 & -0.2505 & -0.2505 & $0.0000 \times 10^{-4}$ \\
0.4 & -0.2768 & -0.2768 & $0.0000 \times 10^{-4}$ \\
0.5 & -0.3059 & -0.3059 & $0.0000 \times 10^{-4}$ \\
0.6 & -0.3381 & -0.3381 & $0.0000 \times 10^{-4}$ \\
0.7 & -0.3737 & -0.3736 & $0.0001 \times 10^{-4}$ \\
0.8 & -0.4130 & -0.4129 & $0.0001 \times 10^{-4}$ \\
0.9 & -0.4564 & -0.4562 & $0.0004 \times 10^{-4}$ \\
1 & -0.5044 & -0.5040 & $0.0016 \times 10^{-4}$ \\
\hline
\end{tabular}

\section{Conclusion}

We have applied the Galerkin's method by using the Bernstein polynomials to find the approximate solution of the linear FIDE of the first order. Using this procedure, the integro-differential form is reduced to solve a system of algebraic equations. Examples have been introduced to demonstrate the validity of the present technique. These examples also exhibit the accuracy and efficiency of the present method.

\section{Acknowledgment}

The authors are very grateful to the anonymous referees and the editor for their guidance and suggestions which greatly improved this paper.

\section{Author's Contributions}

Miloud Moussai: Did the research, analysed and interpreted the results. Prepared the manuscript and responsible for the paper submission.

Lakhdar Chiter: Designed the research plan, supervised and organized the structure of the manuscript and reviewed it. Responsible for the paper corrections.

\section{Ethics}

The corresponding author confirms that the co-author have read and approved the manuscript and no ethical issues involved.

\section{References}

Boyer, R.P. and L.C. Thiel, 2002.Generalized Bernstein polynomials and symmetric functions. Adv. Applied Math., 28: 17-39.

DOI: 10.1006/aama.2002.0765

Golubov, B., A. Efimov and V. Skvortsov, 1991. Walsh Series and Transforms: Theory and Applications. 1st Edn., Springer Netherlands, Dordrecht, ISBN-10: 0792311000, pp: 368.

IdreesBhattia, M. and P. Bracken, 2007. Solutions of differential equations in a Bernstein polynomial basis. J. Comput. Applied Math., 205: 272-280. DOI: 10.1016/j.cam.2006.05.002

Isik, O.R., Z. Güneyin and M. Sezer, 2012. Bernstein series solutions of Pantograph equations using polynomial interpolation. J. Diff. Equ. Applic., 18: 357-374.

DOI: $10.1080 / 10236198.2010 .496456$ 
Singh, A.K., V.K. Singh and O.P. Singh, 2009. The Bernstein operational matrix of integration. Applied Math. Sci., 3: 2427-2436.

Swarup, S., 2007. Integral equations. Krishna Prakashan Media Pvt. Ltd.
Yalçinbaş, S., M. Sezer and H.H. Sorkun, 2009. Legendre polynomial solutions of high-order linear Fredholmintegro-differential equations. Applied Math. Comput., 210: 334-349.

DOI: $10.1016 /$ j.amc.2008.12.090 